\title{
Erratum to: When good advertisement backfires: cleaners get eaten too
}

\author{
J. P. M. Messias $^{1}$ - M. C. Soares ${ }^{1}$
}

Published online: 18 September 2015

(C) Springer-Verlag Berlin Heidelberg 2015

\section{Erratum to: Coral Reefs (2015) DOI 10.1007/s00338-015-1303-1}

The cleaner was captured and released before the occurrence of this predatory event. This methodology has been extensively applied before, without a similar occurrence ever being reported (Soares et al. 2012, 2014; Paula et al. 2015).

\section{References}

Soares MC, Bshary R, Cardoso SC, Côté IM, Oliveira RF (2012) Face Your fears: cleaning Gobies inspect predators despite being stressed by them. PLoS ONE 7(6):e39781. doi:10.1371/journal. pone.0039781
Soares MC, Cardoso SC, Grutter AS, Oliveira RF, Bshary R (2014) Cortisol mediates cleaner wrasse switch from cooperation to cheating and tactical deception. Horm Behav 66:346-350

Paula JR, Messias JPM, Grutter AS, Bshary R, Soares MC (2015) The role of serotonin in the modulation of cooperative behavior. Behav Ecol. doi:10.1093/beheco/arv039

The online version of the original article can be found under doi:10.1007/s00338-015-1303-1.

J. P. M. Messias

joao.messias@cibio.up.pt

1 Centro de Investigação em Biodiversidade e Recursos Genéticos (CIBIO), Universidade do Porto, Campus Agrário de Vairão, 4485-661 Vairão, Portugal 